\title{
Small-Body Encounters Using Solar Sail Propulsion
}

\author{
Gareth W. Hughes* and Colin R. McInnes ${ }^{\dagger}$ \\ University of Glasgow, Glasgow, Scotland G12 8QQ, United Kingdom
}

\begin{abstract}
Small-body, high-energy rendezvous and flyby missions have large $\Delta V$ requirements, which impose extreme demands on the propellant budget for chemical propulsion. Ion propulsion is a viable alternative, but as the number and difficulty of target objectives increases, it then becomes rapidly unattractive due to increasing launch mass and required thruster life. For solar sails, however, propellant mass is not an issue, and so a large $\Delta V$ could be realized without implementing gravitational assists, which prolong mission duration and restrict launch opportunities. High-energy, small-body mission scenarios are analyzed using a parameterized approach to trajectory optimization. Optimal rendezvous trajectories to short-period comets are described. A solar sail mission to comet Wirtanen is presented, which could reduce launch mass by $44 \%$ and trip time by $68 \%$, over the previous baseline Rosetta mission. High-performance sails could be used to permit fast flyby intercepts of newly discovered longperiod comets. Trajectories to comets during past apparitions have been generated, including comet Hale-Bopp, to demonstrate the feasibility of intercepting a new long-period comet using a solar sail at perihelion or orbit node. Earth return or secondary flybys are also investigated. High $\Delta V$, multiple asteroid surveys of up to four main-belt asteroids are investigated. It is shown that if solar sail propulsion were used for the Dawn mission, a launch mass reduction of up to $33 \%$ may be possible.
\end{abstract}

\section{Nomenclature}

$A=$ sail film reflective surface area, $\mathrm{m}^{2}$

$a_{c}=$ characteristic acceleration, $\mathrm{m} \cdot \mathrm{s}^{-2}$ (usually quoted in $\mathrm{mm} \mathrm{s}^{-2}$ )

$c_{i}=$ nonlinear programming equality constraint, where $i=1-n$

$c_{\text {state }}=$ genetic algorithm state constraint weighting coefficient $\left(10^{3}\right.$ for all states)

$c_{t}=$ genetic algorithm transfer time weighting coefficient $\left(10^{-11}\right)$

$J \quad=$ nonlinear programming objective function

$M=$ total spacecraft mass, $\mathrm{kg}$

$m_{p}=$ solar sail payload mass, $\mathrm{kg}$

$n=$ total number of equality constraints ( 3 for flyby, 6 for rendezvous)

$P_{\mathrm{AU}}=$ solar radiation pressure at 1 astronomical unit, $4.56 \times 10^{-6} \mathrm{~N} \cdot \mathrm{m}^{-2}$

$t_{f}=$ terminal time, days

$\begin{array}{ll}t_{f} & =\text { control vector } \\ \boldsymbol{u} & =\end{array}$

$v_{\infty}=$ hyperbolic excess velocity, $\mathrm{km} \mathrm{s}^{-1}$

$\boldsymbol{x}=$ six-element state vector

$\boldsymbol{y}_{i}^{\mathrm{s} / \mathrm{c}}=$ state vector element of spacecraft, where $i=1-n$

$\boldsymbol{y}_{i}^{\mathrm{tgt}}=$ state vector element of target body, where $i=1-n$

$\alpha=$ cone angle

$\Delta V=$ velocity increment for orbit change, $\mathrm{km} \cdot \mathrm{s}^{-1}$

$\delta=$ clock angle

$\eta \quad=$ sail propulsive reflectivity

$\kappa=$ payload mass fraction

$\mu=$ gravitational parameter

$\sigma_{s}=$ sail assembly loading, $\mathrm{kg} \cdot \mathrm{m}^{-2}$ (usually quoted in $\mathrm{g} \cdot \mathrm{m}^{-2}$ )

\section{Introduction}

$\mathbf{L}$ ARGE databases exist of comets whose orbital parameters have been more accurately determined via successive observations,

Received 14 January 2003; revision received 15 July 2003; accepted for publication 17 July 2003. Copyright (C) 2003 by Gareth W. Hughes and Colin R. McInnes. Published by the American Institute of Aeronautics and Astronautics, Inc., with permission. Copies of this paper may be made for personal or internal use, on condition that the copier pay the $\$ 10.00$ per-copy fee to the Copyright Clearance Center, Inc., 222 Rosewood Drive, Danvers, MA 01923; include the code 0022-4650/04 \$10.00 in correspondence with the CCC.

*Postgraduate Student, Department of Aerospace Engineering.

${ }^{\dagger}$ Professor, Department of Aerospace Engineering. such as those of the Bureau des Longitudes in Paris. The material structure of these relics from the early solar system is only beginning to be understood. The comet nucleus is thought to be mainly composed of dust and rock with a significant fraction of ice binding it together. Comets have undertaken a long evolutionary path, and thus, many are pristine examples of matter from the early solar system, condensing from the ancient solar nebula at the same time as when the outer planets and their satellites were in their infancy. ${ }^{1}$ It is now known that many comets form in the Oort Cloud on the edge of interstellar space at 50,000 astronomical units (AU) from the sun. Shorter period comets can originate from the Edgeworth-Kuiper Belt, which lies just beyond Neptune's orbit. There may also be a further repository, the so-called Inner Oort Cloud, which tells us that the source region can be closer to the planetary domain. ${ }^{2}$ Comets are divided, somewhat arbitrarily, into two categories depending on their orbital characteristics. Short-period comets (SPCs), which have periods of less than 200 years originating in the Kuiper Belt, and those of greater than 200 years, the long-period comets (LPCs) born in the Oort Cloud.

The most famous SPC, 1P/Halley, in common with all comets, has a highly inclined and eccentric orbit, but is unusual in that it has a retrograde orbit. In 1976, the NASA Jet Propulsion Laboratory (JPL) conducted a detailed mission study for a planned solar sail rendezvous with Halley on its 1986 inner solar system passage. ${ }^{3}$ The solar sail element was subsequently replaced with solar electric propulsion, but ultimately the mission was cancelled. The ESA spacecraft Giotto encountered Halley in 1986 at a large relative velocity of $68 \mathrm{~km} \cdot \mathrm{s}^{-1}$; nonetheless, valuable information and low-resolution images were acquired. The ESA Rosetta spacecraft missed a January 2003 opportunity to comet wirtanen and has since been retargeted to comet 67P/Churyumov-Gerasimenko, to be launched in February 2004. Numerous studies have been conducted into SPC rendezvous missions enabled by solar electric propulsion. ${ }^{1,4}$

The apparition of bodies classified as LPCs has generated much enthusiasm within the science community. Their eccentricities approach unity, and a few have previously been observed to be extrasolar in origin. However, recent research suggests that almost all LPCs do in fact have elliptical orbits, once their orbits are properly computed via modern methods of estimating the uncertainties. The nongravitational effects due to sublimation of $\mathrm{CO}$ and $\mathrm{H}_{2} \mathrm{O}$ are responsible for small adjustments in the orbital elements to make previously hyperbolic comets become elliptical. ${ }^{5}$ LPCs have undergone a limited number of passages, if any, though the inner solar system. Because of this, they are regarded to be the best preserved bodies in the solar system inasmuch as they have had limited solar 
interaction and consequential cometary activity. ${ }^{6}$ Rendezvous with a newly discovered LPC would be highly impractical, so research conducted so far has involved using ballistic means to flyby at one of the orbit nodes of a new LPC shortly after first discovery. ${ }^{2,6}$ The ill-fated CONTOUR mission launched by NASA on 3 July 2002 was to use successive Earth gravity assists to perform slow flybys of the SPCs Encke then Schwassmann-Wachmann 3, and then was to allow for retargeting to a newly discovered LPC should that exciting opportunity have arisen.

A plethora of planetesimals exist in the solar system. The gravitational forces of Mars and Jupiter prevented planetary accretion of main-belt asteroids, so they can provide evidence of how the solar system formed. A high density of near-Earth objects (NEOs) also abound, which include some of the shorter period comets. Expanding knowledge of these NEOs is vital to catalog the possible threats to Earth, along with obvious scientific connotations. The near-Earth asteroid rendezvous (NEAR)-Shoemaker mission to 433 Eros was a major breakthrough in the understanding of near-Earth asteroids. Rendezvous trajectories have been previously analyzed in the literature using solar sails by Leipold ${ }^{7}$ and also using solar electric propulsion by Colasurdo and Casalino. ${ }^{8}$ Yen also compares solar sail and solar electric propulsion for dual asteroid rendezvous missions. ${ }^{9}$ This paper builds on Yen's previous work, with mass sizing analysis and extension of the target asteroids to two further asteroids. Yen states that, for more than two rendezvous, solar sail propulsion is expected to be superior to solar electric propulsion. Integral to any small-body rendezvous mission is if a solar sail can maintain itself in orbit around the weak gravitational field of an asteroid or comet. This has been treated in the literature, where it was found that certain stable orbits and unstable hover points exist. ${ }^{10,11}$

\section{Solar Sail Propulsion}

Solar sail propulsion has been postulated since the early 20th century, first envisaged by the pioneers of Soviet astronautics, Tsiolkovsky and Tsander (see Ref. 12). Much work was conducted for a comet Halley rendezvous mission, during the late 1970s at JPL, both in hardware and trajectory design. Indeed, Sauer has been instrumental in optimizing a wide range of heliocentric trajectories. ${ }^{13}$ Forward has also contributed much to the field in recent years, particularly with regard to unique non-Keplerian orbits. ${ }^{14}$ The wellfounded physical principle of solar sail propulsion is that solar photons have momentum, which is imparted to the gossamer sail film and then, in reflection, also exert a reaction force producing a thrust directed normal to an ideal sail surface. The elimination of propellant means that the velocity increment can be extremely large for the extended mission tours proposed. Solar sailing is strongly established in NASA and ESA technology roadmaps. Recent technological advances include development of $1.5-\mu \mathrm{m}$ CP1 polyimide film. ${ }^{15}$ ESA and the DLR, German Aerospace Research Center, conducted a $20 \times 20 \mathrm{~m}$ square sail ground deployment test in 1999. In addition, privately funded orbital demonstrations, by the Planetary Society and by Team Encounter, are scheduled over the next 2 years. Many small-body missions described utilize conventional or solar electric propulsion with a number of gravity assist maneuvers, in an attempt to minimize the propellant required for the large $\Delta V$ necessary to obtain highly inclined and eccentric orbits. There are potentially huge benefits of using the large $\Delta V$ capability of solar sail propulsion because propellant mass is irrelevant. Therefore, propellant mass-conserving, gravitational assists are not necessarily required, and the sail can perform a powered arc or spiral directly to the target body.

For an ideal sail, the parameter defining the thrust is known as the characteristic acceleration $a_{c}$, the solar radiation pressure induced acceleration at $1 \mathrm{AU}$ with the sail normal oriented along the sunspacecraft line. The characteristic acceleration is related to the local photon pressure at $1 \mathrm{AU}$, the sail film reflective surface area, and total spacecraft mass, by

$$
a_{c}=2 \eta P_{\mathrm{AU}} A / M
$$

Because photon pressure is inversely proportional to the square of the distance from the sun, of course, the acceleration also di- minishes. Solar sails are, therefore, highly effective within the inner solar system. ${ }^{15}$ For trajectory optimization, the solar sail has been assumed to be an ideal, flat, perfectly reflecting surface. In reality, a fraction of the incident radiation will be absorbed due to nonperfect reflectivity. (Aluminum has a reflectivity of $85-95 \%$.) To reduce thermal loading, a proportion of this energy needs to be reradiated from the back surface of the sail using a highly emissive coating such as chromium (emissivity of $64 \%$ ). A real sail will also have wrinkles, causing nonspecular reflective effects. The photon pressure will cause the sail to billow, however well designed the supporting structure may be. These factors have the effect of reducing the available thrust produced on a particular sail area and cause the thrust orientation to become offset from the sail normal, the centerline effect. The imperfect effects mentioned will not be considered in this initial survey of mission opportunities, except for mass sizing purposes.

\section{Method}

\section{Control Representation}

The sail thrust vector direction has been defined by two angles to cover completely the outward hemisphere of allowable orientations. These control angles are the cone angle, $\alpha \in[-\pi / 2, \pi / 2]$, between the sail normal and the sun line, and the clock angle, $\delta \in[0, \pi]$, between the projection of the sail normal and some reference direction onto a plane normal to the sun line. ${ }^{12}$ A direct, parameter optimization scheme was implemented with the controls (cone and clock angles) specified at discrete nodes at the segment boundaries, equally spaced in time between zero and the terminal time. The controls were characterized across each time segment by linear interpolation between the nodes. As the number of nodes was increased, a close approximation to a continuous profile was achieved. Problems requiring more revolutions, or more rapid control variation, clearly needed more segments. In this paper 50 segments (51 nodes) was the upper limit.

\section{Optimization Method}

A hybrid method of trajectory optimization has been employed, similar to that used to investigate transfers to non-Keplerian orbits. ${ }^{16}$ The trajectory optimization problem is to select the variables that minimize the transfer time (objective function), while satisfying the endpoint boundary conditions (constraints). There are six endpoint Cartesian state vector constraints for rendezvous and three for flyby, in addition to any problem specific constraints. Therefore, the objective function must be minimized, subject to the equality constraints. This can be formulated as a general optimal control problem, where the objective function has the form

$$
J\left[x\left(t_{f}\right), \boldsymbol{u}, t_{f}\right]=f\left[x\left(t_{f}\right), \boldsymbol{u}, t_{f}\right]+\int_{0}^{t_{f}} g[x(t), \boldsymbol{u}, t] \mathrm{d} t
$$

This was transcribed to a nonlinear programming (NLP) problem, solved using NPSOL 5.0, a FORTRAN77 package based on sequential quadratic programming (SQP). SQP employs a quasi-Newton approximation to the Karush-Kuhn Tucker conditions of optimality, resulting in a subproblem of minimizing a quadratic approximation to the function of Lagrange multipliers incorporating the objective and constraints (see Ref. 16). Gradient evaluation was approximated by finite differences.

Unfortunately these gradient-based, deterministic, local-search NLP solvers require an initial guess of the optimization variables to ensure that a feasible solution is obtained. For the majority of the trajectories, successive forward integrations were performed and trajectories visualized until an estimated control profile produced a spacecraft endpoint state vector that was close enough to the target endpoint state vector to allow NPSOL to find an optimal solution. In a number of cases where engineering insight could not be used, the global search properties of a genetic algorithm were employed to generate the initial guess. The specifics of this algorithm are covered by Refs. 16 and 17 with the fitness function similar to that used by 
Bader. ${ }^{18}$ The constraints are incorporated into the fitness function as penalty terms:

$$
\text { fitness }_{\mathrm{GA}}=1 / J_{\mathrm{GA}}=1 /\left[c_{t} t_{f}^{2}+\sum_{i=1}^{n} c_{\text {state }}\left(\boldsymbol{y}_{i}^{\mathrm{s} / \mathrm{c}}-\boldsymbol{y}_{i}^{\mathrm{tgt}}\right)^{2}\right]
$$

\section{Mathematical Model}

The optimization proceeds by explicitly integrating the variational equations of the modified equinoctial orbital elements at each function evaluation, using an adaptive step-size, variable-order, Adams-Moulton-Bashforth method (see Ref. 19). Two-body dynamics were modeled with the primary body, the sun, considered to be a point source of radiation. The single perturbation considered was solar photon pressure, and the solar wind is negligible in comparison. The state vectors of the small bodies were obtained by specifying the first five classical orbit elements, the mean motion, and time of perihelion passage. With time as the independent variable, the true anomaly could be determined. At Earth departure, there was zero hyperbolic excess velocity for all trajectories, so that at the sphere of influence of the Earth the spacecraft was assumed to have parabolic escape conditions.

\section{SPC Wirtanen Rendezvous}

The ESA spacecraft, Rosetta is an ESA Horizon (2000) cornerstone mission. The objective of this mission was to rendezvous with the SPC 46P/Wirtanen. This opportunity has been missed, and a new target of 67P/Churyumov-Gerasimenko has been identified; however, this paper will concentrate on the original Wirtanen mission for comparison purposes. Noted that solar sails are not restricted by launch windows, which allows for more flexibility in target selection. This ballistic mission was to have a total trip time of almost 9 years and was to use one Mars and two Earth gravity assists to reduce chemical propellant mass requirements. This extremely long trip time would have also been offset by conducting science observation during on-route flybys of the mainbelt asteroids, Otawara and Siwa. Departure was scheduled for 12 January 2003 from the Kourou spaceport aboard an Ariane 5 launch vehicle, which was to provide $3.4 \mathrm{~km} \cdot \mathrm{s}^{-1}$ of hyperbolic excess velocity. The maximum (wet) launch mass was 2.95 tonnes. The subsystems, lander, and scientific payload account for $878.3 \mathrm{~kg}$, in addition to 2.07 tonnes of propellant, engine, and tanks. This enormous quantity of propellant is necessary for a chemical mission to a high-energy target with such a large spacecraft. To replace the primary propulsion system with a solar sail could in principle reduce the launch mass and enable a cheaper launch vehicle to be used. Solar sail trajectory and mission analysis was, therefore, conducted for this baseline mission.

Rendezvous with a comet in a highly eccentric orbit is difficult to achieve, so that the formulation of the initial guess is easier with a low number of heliocentric revolutions, enabled by high-acceleration capability. Initially, the characteristic acceleration was set at $5.0 \mathrm{~mm} \cdot \mathrm{s}^{-2}$. This acceleration is representative of high-performance sails, such as those considered by JPL for Heliopause missions..$^{20}$ In the first instance, the launch date was fixed at 1 October 2007. The transfer times are shown in Table 1 for increasing control node resolution. These solutions were then used as the initial control estimate for the launch date variable optimizations. For 50 segments ( 51 nodes), the solution found was 205.0 days for an optimal launch date of 17 October 2007. The characteristic acceleration was then decreased by incremental solution feedback

Table 1 Wirtanen rendezvous times of $5.0 \mathrm{~mm} \cdot \mathrm{s}^{-2}$ as function of control resolution

\begin{tabular}{lcc}
\hline $\begin{array}{l}\text { Number of control } \\
\text { segments }\end{array}$ & $\begin{array}{c}\text { Number of } \\
\text { control nodes }\end{array}$ & $\begin{array}{c}\text { Transfer time, } \\
\text { days }\end{array}$ \\
\hline 5 & 6 & 231.0 \\
10 & 11 & 228.4 \\
20 & 21 & 211.9 \\
50 & 51 & 208.1 \\
\hline \hline
\end{tabular}

Table 2 Wirtanen rendezvous times against characteristic acceleration

\begin{tabular}{lcc}
\hline $\begin{array}{l}\text { Characteristic } \\
\text { acceleration, } \mathrm{mm} \cdot \mathrm{s}^{-2}\end{array}$ & $\begin{array}{c}\text { Optimal launch } \\
\text { date }\end{array}$ & $\begin{array}{c}\text { Transfer time, } \\
\text { days }\end{array}$ \\
\hline 5.0 & 19 Oct. 2007 & 208 \\
4.0 & 14 Oct. 2007 & 237 \\
3.0 & 1 Sept. 2007 & 279 \\
2.0 & 3 Oct. 2007 & 490 \\
1.0 & 15 Dec. 2006 & 1043 \\
\hline \hline
\end{tabular}

(adjusting the acceleration in small increments, passing the solution back as the initial guess for the next optimization) along with the launch date variable. Appropriate bounds were selected for the launch date to ensure that the solution did not stray too close to the boundaries, which caused algorithmic convergence problems. The results are shown in Table 2 . The trajectory with a characteristic acceleration of $1.0 \mathrm{~mm} \cdot \mathrm{s}^{-2}$ is shown in Fig. 1, where the trip time of 2.86 years is a $68 \%$ reduction over the 8.9 -year, ballistic trip time. The temporal evolution of the semimajor axis and inclination is shown in Fig. 2. It can be seen that maximum rate of change of inclination occurred during the first 300 days of flight, where the solar photon flux was higher. After this initial cranking phase, semimajor axis boost occurs, and Wirtanen is reached shortly before its aphelion.

Using the Rosetta spacecraft mass (minus the propellant and propulsion components) of $878.3 \mathrm{~kg}$, some analysis was conducted into the required sail dimensions to enable this trajectory. Once total spacecraft mass (including sail structure) was determined, then a launch vehicle could be selected. The solar sail technology level is defined by the sail assembly loading. The sail assembly loading is the usable areal density of the sail film, booms, adhesives, associated tensioning and deployment structures, and mechanisms of which the total sail propulsion component is comprised. Rapid technological development is currently taking place for upcoming demonstrator and operational missions. The Team Encounter mission will use $0.9-\mu \mathrm{m}$ film of $85 \%$ propulsive reflectivity, with linear boom densities as low as $14.1 \mathrm{gm}^{-1}$, which will enable a highly attractive sail assembly loading of $3.4 \mathrm{~g} \cdot \mathrm{m}^{-1}$ (Ref. 21). However, due to some growth in boom mass, recent calculations suggest this will increase to $3.56 \mathrm{gm}^{-2}$. The total spacecraft mass is obtained from Eq. (1) as

$$
M=2 \eta P_{\mathrm{AU}} A / a_{c}
$$

The nonperfect sail efficiency factor represents the percentage of photons reflected by the sail film, assumed to be $85 \%$. The payload mass (subsystems and scientific payload) is, thus, defined by

$$
m_{p}=M-\sigma_{s} A
$$

The payload mass fraction can, therefore, be obtained from

$$
\kappa=m_{p} / M=1-a_{c} \sigma_{s} / 2 \eta P_{\mathrm{AU}}
$$

The total launch mass is, thus, $M=m_{P} / \kappa$. The total reflective surface area of the sail can be obtained from

$$
A=\left(M-m_{p}\right) / \sigma_{s}
$$

Table 3 shows the variation of launch mass with sail assembly loading, with the resulting square sail side length. If a sail were fabricated with the same performance of the Encounter sail $\left(\sigma_{s}=3.56 \mathrm{~g} \cdot \mathrm{m}^{-2}\right)$, then the 458 by $458 \mathrm{~m}$ sailcraft would weigh $1624 \mathrm{~kg}$. This represents a $44 \%$ reduction in launch mass, $v_{\infty}=0$, over the original Rosetta mission, $v_{\infty}=3.4 \mathrm{~km} \cdot \mathrm{s}^{-1}$, and would mean that a smaller, cheaper launch vehicle could be used, such as a Soyuz ST-Fregat or Delta 2 7925-10. The solar sail could also be used to escape from geosynchronous transfer orbit (GTO) and then reach Wirtanen in 2.86 years, much less than the original Rosetta 8.9-year trip time. 


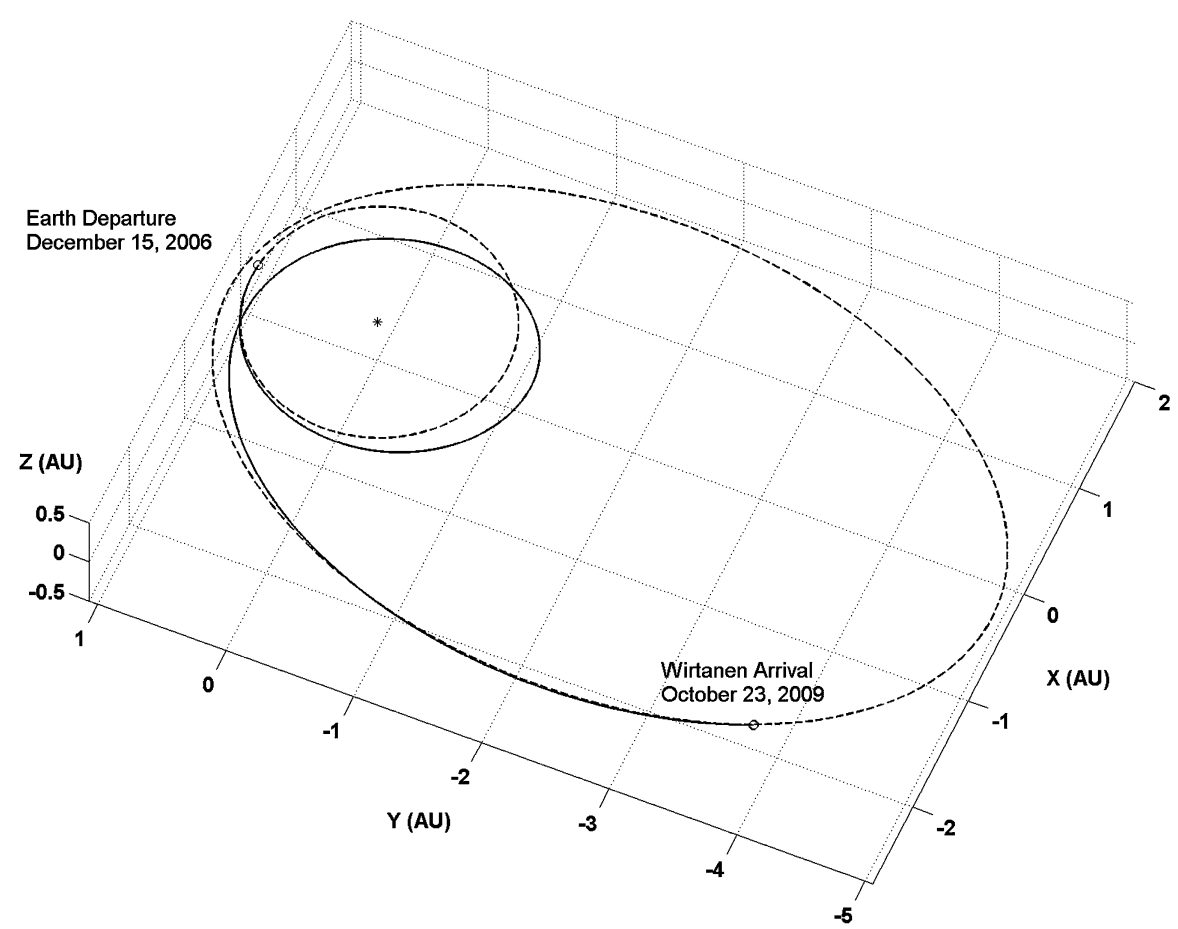

Fig. 1 Wirtanen rendezvous trajectory of $1.0 \mathrm{~mm} \cdot \mathrm{s}^{-2}$.

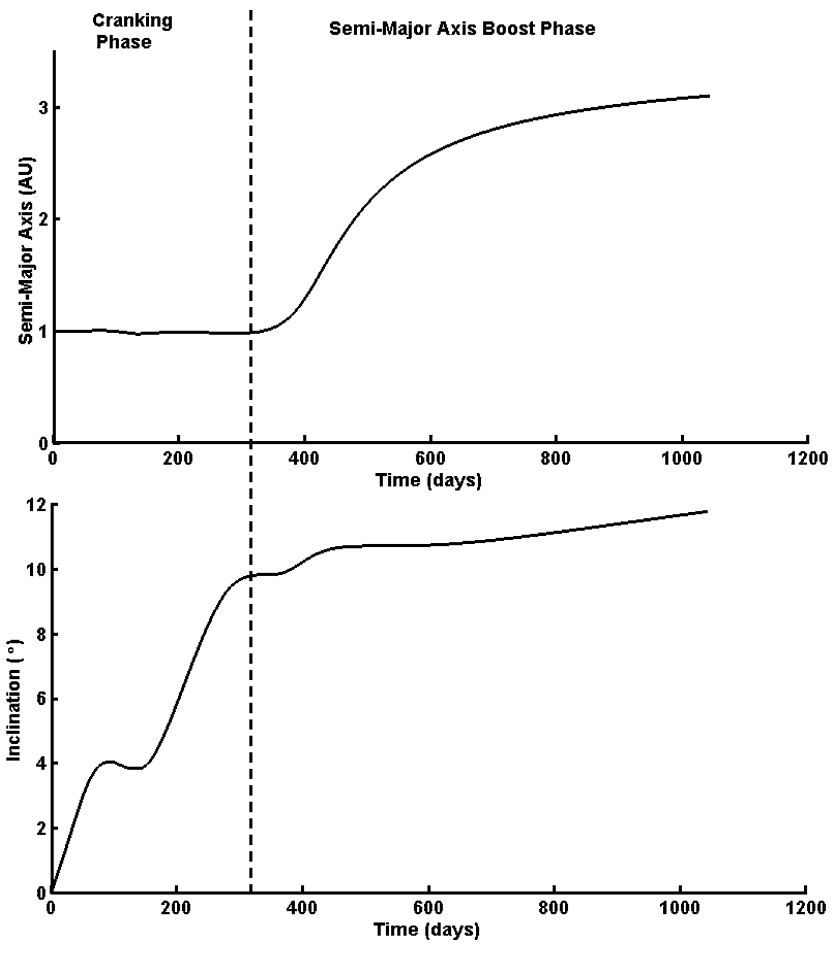

Fig. 2 Wirtanen rendezvous orbital element evolution.

\section{LPC Flybys}

An analysis using high-performance sails was conducted to permit fast flyby intercepts of newly discovered LPCs. Because of the nature of this problem, previous comet apparitions were adopted to demonstrate the feasibility of a late launch to intercept quickly a new LPC using a solar sail. Because the time between discovery of the new LPC Hale-Bopp and perihelion passage was less then 2 years, this left a very short time span for spacecraft preparation, orbit planning, and launch/operational phases. Therefore, for future new LPC apparitions, it is envisaged that the stowed sail would hibernate in GTO or geostationary Earth orbit (GEO) with a small
Table 3 Sailcraft sizing for $1.0 \mathrm{~mm} \cdot \mathrm{s}^{-2}$ Wirtanen rendezvous mission with Rosetta payload

\begin{tabular}{lccc}
\hline \hline $\begin{array}{l}\text { Sail assembly } \\
\text { loading } \sigma_{s}, \mathrm{~g} \cdot \mathrm{m}^{-2}\end{array}$ & $\begin{array}{c}\text { Payload mass } \\
\text { fraction } \kappa\end{array}$ & $\begin{array}{c}\text { Launch mass, } \\
\mathrm{kg}\end{array}$ & $\begin{array}{c}\text { Side length, } \\
\mathrm{m}\end{array}$ \\
\hline 2 & 0.742 & 1183.7 & 390.8 \\
3 & 0.613 & 1432.8 & 430.0 \\
3.56 & 0.541 & 1623.4 & 457.5 \\
4 & 0.484 & 1814.7 & 483.8 \\
5 & 0.355 & 2474.1 & 564.9 \\
6 & 0.226 & 3886.3 & 708.1 \\
7 & 0.097 & 9054.6 & 1080.8 \\
\hline \hline
\end{tabular}

science payload. When a target is sighted, the sail would deploy, and the spacecraft could rapidly escape from Earth orbit to reach the new LPC.

\section{Hale-Bopp Perihelion Flyby}

As an example of the type of new LPC that could appear, the case of the 1995 apparition of the comet C/1995 O1/Hale-Bopp was considered. Because of the inclination and orbit geometry, the perihelion is displaced quite far above the ecliptic plane. Therefore, initially, a high-performance sail was utilized with a characteristic acceleration of $5.0 \mathrm{~mm} \cdot \mathrm{s}^{-2}$. The optimization problem was then to minimize the transfer time such that the launch date was the time of perihelion passage minus the transfer time. The final solution established by NPSOL was 208.8 days, resulting in a launch date of 4 September 1996. The trajectory is shown in Fig. 3. The characteristic acceleration was then decreased by the incremental feedback method. The number of linear interpolation nodes was increased to 21 nodes, with 20 trajectory segments. These results are shown in Table 4. It was then attempted to minimize the transfer time and the relative velocity at flyby. NPSOL only permits for one objective function and so the relative velocity at flyby was encoded as an extra inequality constraint. The upper limit on this inequality was specified so that NPSOL would force the additional variable to be below the limit. This upper limit was decreased in increments while passing the solution back each time as the next initial guess. This was performed for a characteristic acceleration of $2.0 \mathrm{~mm} \cdot \mathrm{s}^{-2}$, with the unconstrained relative velocity being $58.6 \mathrm{~km} \cdot \mathrm{s}^{-1}$. The results are 
shown in Table 5. The final relative velocity was always equal to the upper bound set for each transfer. The trajectory for the $36 \mathrm{~km} \cdot \mathrm{s}^{-1}$ flyby is shown in Fig. 4. It can be seen that the vertical component of the spacecraft and comet velocity is closely matched.

\section{Hale-Bopp Descending Node Flyby}

High-performance sails are required to enable the high accelerations necessary to intercept many comets at perihelion. It would be easier to intercept a comet at one of its orbit nodes because the transfer trajectory can remain within the ecliptic plane; inclination changes are not necessary. The spacecraft can also be more readily returned to the Earth if required. Other new comets, subsequently discovered, could then also be intercepted at one of their nodes; the inclination of the heliocentric parking orbit plane is, thus, standardized.

The perihelion flyby optimization code was adjusted to perform flyby at a user-specified node. (The nearest node to Earth orbit was

Table 4 Hale-Bopp perihelion flyby times

\begin{tabular}{lc}
\hline \hline $\begin{array}{l}\text { Characteristic acceleration, } \\
\mathrm{mm} \cdot \mathrm{s}^{-2}\end{array}$ & $\begin{array}{c}\text { Transfer time, } \\
\text { days }\end{array}$ \\
\hline 5.0 & 208.7 \\
4.0 & 251.7 \\
3.0 & 387.8 \\
2.0 & 537.1 \\
1.64 & 665.2 \\
\hline \hline
\end{tabular}

Table 5 Minimized relative velocity of 2.0-mm $\cdot \mathrm{s}^{-2}$ Hale-Bopp perihelion flyby times

\begin{tabular}{lc}
\hline \hline $\begin{array}{l}\text { Minimized relative velocity } \\
\text { at flyby, } \mathrm{km} \cdot \mathrm{s}^{-1}\end{array}$ & $\begin{array}{c}\text { Transfer time, } \\
\text { days }\end{array}$ \\
\hline 55 & 540.3 \\
50 & 556.9 \\
45 & 585.3 \\
40 & 629.7 \\
37 & 652.1 \\
36 & 661.3 \\
\hline \hline
\end{tabular}

always taken.) The descending node of Hale-Bopp was the easiest node to reach because it was the closest to the Earth's orbit, and so it was selected for flyby. The characteristic acceleration was initially set at $5.0 \mathrm{~mm} \cdot \mathrm{s}^{-2}$, and the number of control nodes was set at 21 (20 segments). The optimal solution was 201.5 days, departing 17 October 1996. The relative flyby velocity was less than for the perihelion flyby case at $46.6 \mathrm{~km} \cdot \mathrm{s}^{-1}$, as expected, and the trajectory is shown in Fig. 5. The trajectory has an inward kink, just before flyby, because of the excessive acceleration capability available, the outward thrust constraint, and the comet nodal passage phasing with the Earth ephemeris. The final semimajor axis of the spacecraft was $0.6156 \mathrm{AU}$, and the eccentricity was 0.9951 . If the spacecraft remained in this orbit, then the perihelion distance would be $449,000 \mathrm{~km}$, which is less than the sun's radius $(695,000 \mathrm{~km})$, and solar surface impact would occur. To avoid this, the sail should be steered toward the next new comet, or into a safer parking orbit, or it could be returned to Earth for potential dust sample return or telemetry download. Note that the postflyby options available are broad because the solar sail has a near infinite $\Delta V$ capability. The Earth return trajectory was optimized because it had the most easily defined criteria. No minimization of flyby relative velocity was implemented; thus, in general, the higher the characteristic acceleration was, the higher the instantaneous flyby eccentricity. Unfortunately, once the solar sail was on a hyperbolic orbit, the optimizer could not generate a feasible solution to allow recapture into a closed orbit. Therefore, it was considered to be easier to use a lower acceleration for flyby and Earth return because the flyby eccentricity would be lower. The characteristic acceleration was, therefore, decreased with the resulting flyby times shown in Table 6 . A characteristic acceleration of $2.0 \mathrm{~mm} \cdot \mathrm{s}^{-2}$ was selected for the flyby and return trajectory. The relative velocity at flyby in this case was $50.4 \mathrm{~km} \cdot \mathrm{s}^{-1}$. The perihelion distance of the instantaneous spacecraft flyby trajectory was 34 million $\mathrm{km}$ or 48.9 solar radii, so that if the sail was jettisoned the spacecraft would not, in principle, impact the sun. For the return trajectory, the optimal solution was of 261.4 days, starting from the flyby initial conditions and returning to Earth sphere of influence with zero hyperbolic excess velocity. The arrival date back at the Earth was 22 January 1998, and the total trip time for flyby and return was 531.7 days (1.46 years). The total trip time is, therefore, the minimum turnaround time for each new comet intercept with

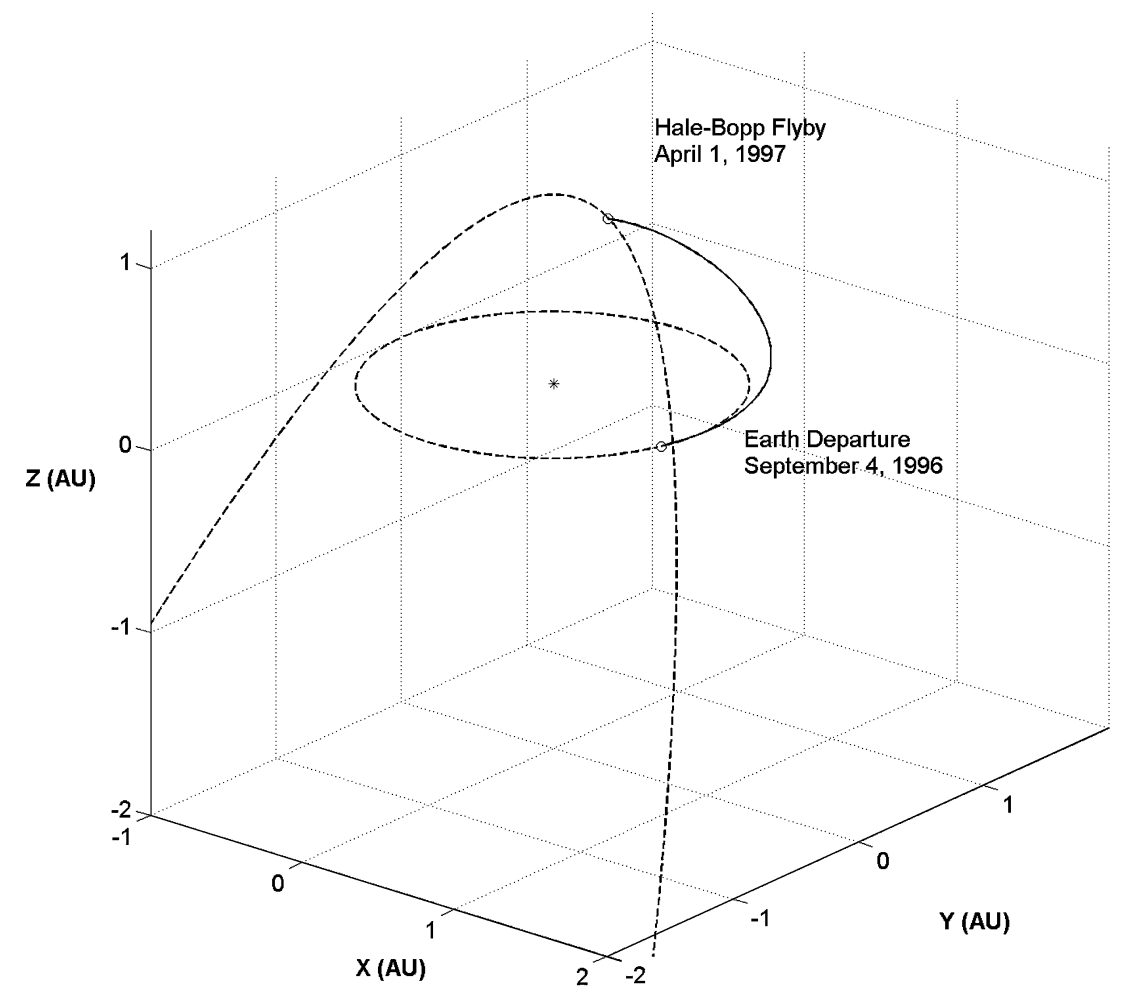

Fig. 3 Hale-Bopp perihelion flyby of $5.0 \mathrm{~mm} \cdot \mathrm{s}^{-2}$. 


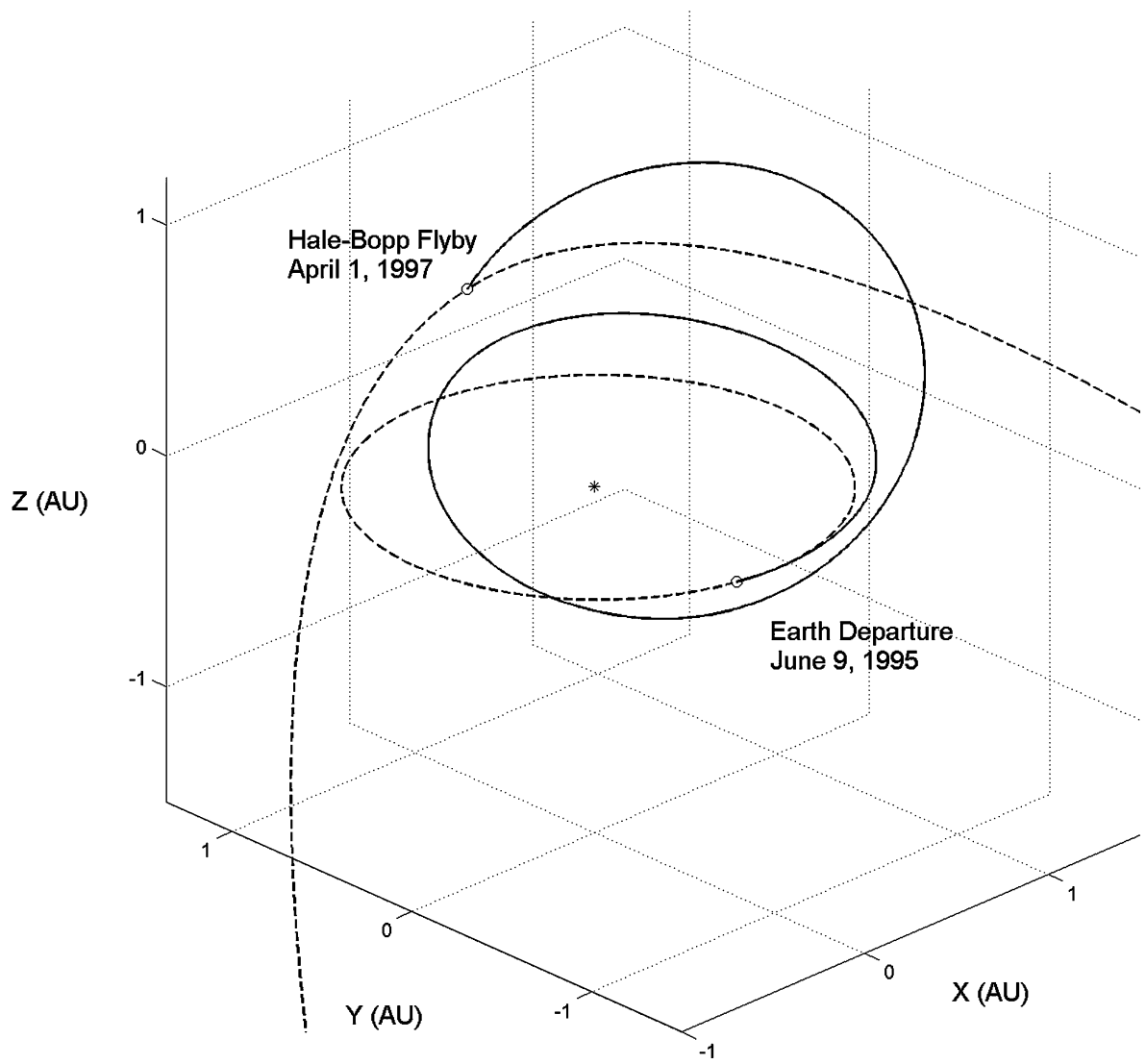

Fig. 4 Hale-Bopp minimized relative velocity flyby of $2.0 \mathrm{~mm} \cdot \mathrm{s}^{-2}$.

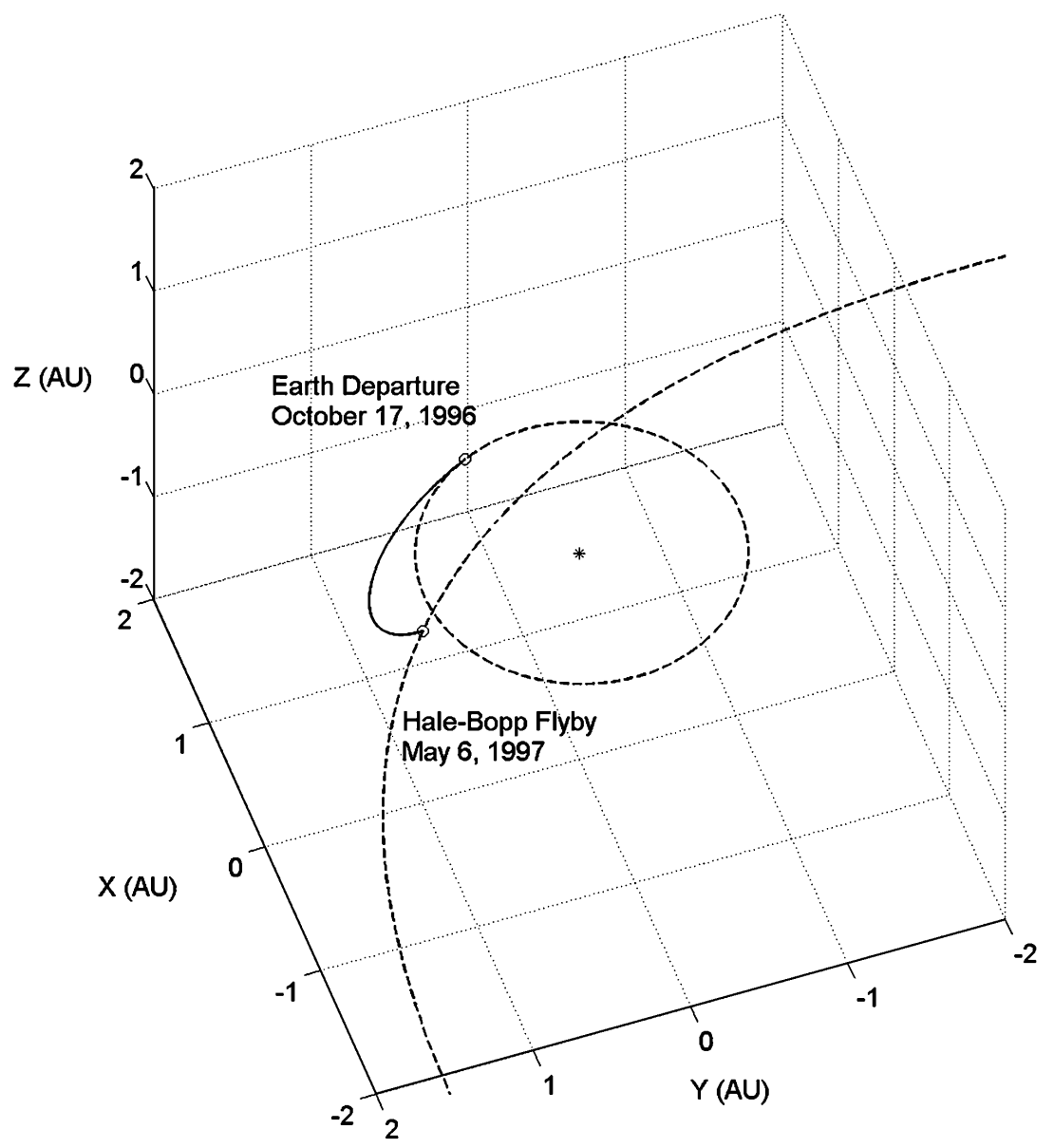

Fig. 5 Hale-Bopp descending node flyby of $5.0 \mathrm{~mm} \cdot \mathrm{s}^{-2}$. 
Earth return for this characteristic acceleration. The entire roundtrip trajectory is shown in Fig. 6. A catalog of additional comet nodal flyby trajectories has been generated to numerous comets as presented in Table 7.

\section{Dual Comet Flyby}

An alternative extension of the Hale-Bopp nodal flyby mission could have been to continue on to flyby another LPC at one of its

Table 6 Hale-Bopp descending node flyby times

\begin{tabular}{lc}
\hline $\begin{array}{l}\text { Characteristic acceleration, } \\
\mathrm{mm} \cdot \mathrm{s}^{-2}\end{array}$ & $\begin{array}{c}\text { Transfer time, } \\
\text { days }\end{array}$ \\
\hline 5.0 & 201.5 \\
4.0 & 217.6 \\
3.0 & 239.2 \\
2.0 & 270.3 \\
1.0 & $328.7^{\mathrm{a}}$ \\
0.5 & $404.7^{\mathrm{a}}$ \\
0.2 & $664.4^{\mathrm{a}}$ \\
\hline \hline
\end{tabular}

${ }^{\mathrm{a}}$ Used 51 control nodes instead of 21. nodes. In principle, the near infinite $\Delta V$ capability could enable several new LPCs to be intercepted. The comet C/1997 D1/Mueller was discovered on 17 February 1997. The discovery date is less than three months before the Hale-Bopp encounter occurred, with the sail enroute to flyby. In this analysis, Mueller was intercepted at its descending node using the same characteristic acceleration of $2.0 \mathrm{~mm} \cdot \mathrm{s}^{-2}$ as before. The transfer time was constant because it was just the difference in the date between Hale-Bopp and Mueller nodal descent. Therefore, Mueller nodal flyby occurred 146.1 days after Hale-Bopp nodal flyby, on 29 September 1997. The trajectory is shown in Fig. 7. The relative velocity at Hale-Bopp was $50.4 \mathrm{~km} \cdot \mathrm{s}^{-1}$ and was $57.6 \mathrm{~km} \cdot \mathrm{s}^{-1}$ at Mueller. At Mueller flyby, the spacecraft reached a hyperbolic escape trajectory due to the close solar photonic assist at 28.3 solar radii. This close approach to the sun would pose serious thermal loading problems on the sail structure, although high-emissivity rear coatings can control sail film temperature. The heliocentric velocity was $45 \mathrm{~km} \cdot \mathrm{s}^{-1}$, which is considerably greater than the $28.1 \mathrm{~km} \cdot \mathrm{s}^{-1}$ solar system escape velocity, at Mueller flyby. Note that the Heliopause (at $100 \mathrm{AU}$ ) could then be reached in 12 years with the outer Heliopause boundary (200 AU), the edge of interstellar space, attained in 24 years. Multiple flybys and the

Table 7 Example LPC nodal flyby times

\begin{tabular}{lcccc}
\hline \hline Comet & $a_{c}, \mathrm{~mm} \cdot \mathrm{s}^{-2}$ & Node $^{\mathrm{a}}$ & Transfer time, days & Earth return time, days $^{\mathrm{b}}$ \\
\hline C/1995 O1 & 2.0 & $\mathrm{D}$ & 270.3 & 261.4 \\
Hale-Bopp & 2.0 & $\mathrm{~A}$ & 240.0 & $\mathrm{H}$ \\
C/1995 Y1 & 2.0 & $\mathrm{~A}$ & 237.8 & $\mathrm{H}$ \\
Hyakutake & & $\mathrm{D}$ & 589.2 & $\mathrm{H}$ \\
C/1999 T1 & 5.0 & $\mathrm{~A}$ & 192.8 & 283.9 \\
McNaught-Hartley & 2.0 & $\mathrm{~A}$ & 268.9 & 244.5 \\
C/1999 F1 & & & \\
Catalina & & & \\
C/1999 N2 & 2.0 & &
\end{tabular}

${ }^{a}$ Descending (D) and ascending (A). ${ }^{\mathrm{b}}$ Hyperbolic escape at flyby (H).

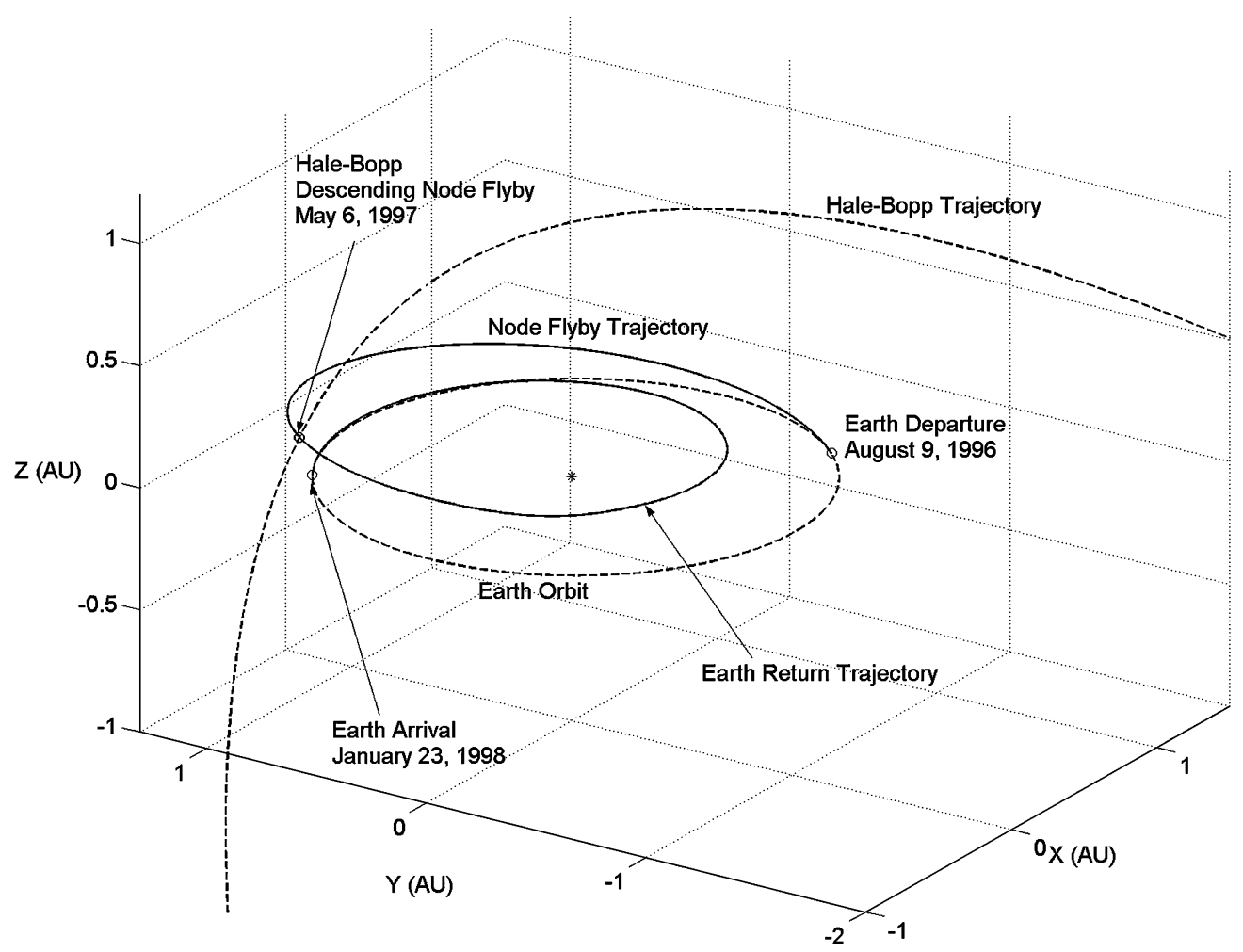

Fig. 6 Hale-Bopp flyby and return trajectory of $2.0 \mathrm{~mm} \cdot \mathrm{s}^{-2}$. 
Table 8 Sailcraft sizing for $0.362-\mathrm{mm} \cdot \mathrm{s}^{-2}$ Hale-Bopp nodal flyby

\begin{tabular}{|c|c|c|c|c|}
\hline \multirow{2}{*}{$\begin{array}{l}\text { Sail assembly } \\
\text { loading } \sigma_{s}, \mathrm{~g} \cdot \mathrm{m}^{-2}\end{array}$} & \multirow{2}{*}{$\begin{array}{l}\text { Payload mass } \\
\text { fraction } \kappa\end{array}$} & \multicolumn{3}{|c|}{ Sail side length, $\mathrm{m}$} \\
\hline & & 20-kg Payload mass & 50-kg Payload mass & 100-kg Payload mass \\
\hline 5 & 0.767 & 34.9 & 55.2 & 78.1 \\
\hline 10 & 0.533 & 41.9 & 66.2 & 93.6 \\
\hline 15 & 0.300 & 55.8 & 88.3 & 124.9 \\
\hline
\end{tabular}

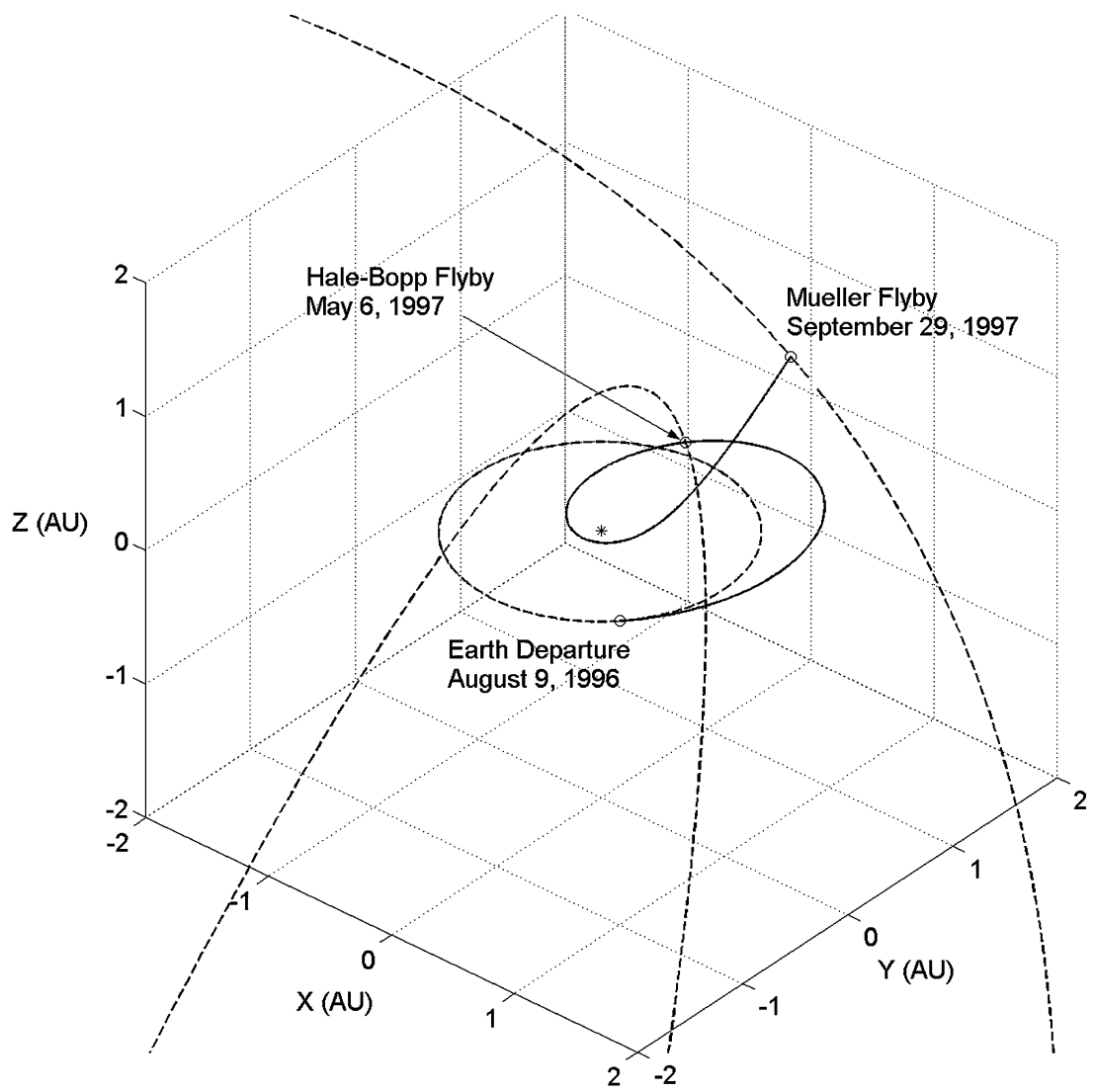

Fig. 7 Earth-Hale-Bopp-Mueller dual comet nodal flyby.

potential for solar system escape demonstrate the great versatility of solar sail propulsion.

\section{Hale-Bopp Opportunity Mission Analysis}

More extensive analysis was conducted into the earlier HaleBopp nodal flyby case, initially for a characteristic acceleration of $2 \mathrm{~mm} \cdot \mathrm{s}^{-2}$. The solar sail escape phase from the Earth's sphere of influence was modeled using analytical control laws, taking into account geopotential perturbations, lunar and solar gravity, and Earth and lunar shadow with umbra and penumbra effects. ${ }^{22}$ After hibernation of the stowed sail in GTO, the sail was deployed on 8 June the escape spiral duration was 59 days, reaching escape conditions, $v_{\infty}=0$, on 6 August. The interception phase was then initiated on 9 August 1996. The discovery date of Hale-Bopp was 23 July 1995 so that this would have left 321 days of contingency (difference between discovery and sail deployment). It would be envisaged that the spacecraft and sail could hibernate in GTO or GEO until required by the appearance of a new LPC. The escape times from GEO, for the range of characteristic accelerations used in the interception phase, were obtained and added to the heliocentric transfer times. First discovery, launch date contingency time against characteristic acceleration is plotted in Fig. 8. Zero contingency occurred for a characteristic acceleration of $0.362 \mathrm{~mm} \cdot \mathrm{s}^{-2}$. A mass and area sizing investigation was conducted for a sail exhibiting this acceleration magnitude. Although a perfect sail was assumed for optimization purposes, an imperfect sail efficiency of $85 \%$ was chosen for the mass sizing exercise. Square sail side length, as a function of sail assembly loading and payload mass, is shown in Table 8. A pay-

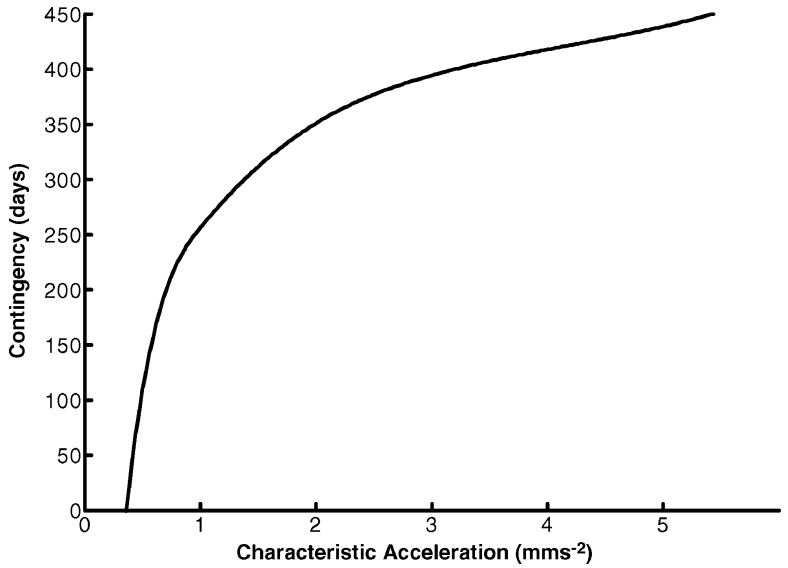

Fig. 8 Launch date contingency against characteristic acceleration for Hale-Bopp nodal encounter.

load mass of $100 \mathrm{~kg}$ is representative of small science missions, and for a payload mass of $20 \mathrm{~kg}$, we would envisage microsatellite technology with a small camera and stripped-down payload. The sail/spacecraft composite with a conservative assembly loading of $15 \mathrm{~g} \cdot \mathrm{m}^{-2}$ could have delivered a payload of $50 \mathrm{~kg}$ using square sail dimensions of less than $90 \times 90 \mathrm{~m}$ and with a total mass of $167 \mathrm{~kg}$. This total mass is within the GEO auxiliary payload capability of a Delta 4 class launcher. 


\section{Asteroid Survey}

Multiple asteroid rendezvous missions have immense $\Delta V$ requirements. Missions to rendezvous with more than two or three target objects make chemical or even solar electric propulsion much less viable as candidate transfer methods. ${ }^{9}$ The structural and material endurances are the only limiting factors dictating the number and range of asteroids the solar-sail-propelled spacecraft can encounter throughout its lifetime. Solar electric propulsion has been adopted as the primary propulsion system for NASA's Discovery class mission, Dawn. Dawn is a dual asteroid rendezvous mission scheduled for launch on 27 May 2006 aboard a Delta $27925 \mathrm{H}$ (2925H) (Ref. 23). The objective of this mission is to rendezvous with inner main-belt asteroids, Vesta and Ceres. Dawn takes 4.2 years to reach Vesta from Earth and, following 11 months in orbit about Vesta, reaches Ceres in 3.1 years. Solar electric propulsion is used, with the 3 NSTAR xenon engines processing $400 \mathrm{~kg}$ of propellant via a $7.5-\mathrm{KW} \mathrm{GaAs}$ solar array. The total wet launch mass is $1108 \mathrm{~kg}$. Therefore, the dry mass (including propulsion) is $708 \mathrm{~kg}$. Propulsion system sizing is taken from Ref. 4 and assumes that two power processing units, a

Table 9 Sailcraft sizing for $1.0-\mathrm{mm} \cdot \mathrm{s}^{-2}$ asteroid rendezvous mission with Dawn payload

\begin{tabular}{lccc}
\hline \hline $\begin{array}{l}\text { Sail assembly } \\
\text { loading } \sigma_{s}, \mathrm{~g} \cdot \mathrm{m}^{-2}\end{array}$ & $\begin{array}{c}\text { Payload mass } \\
\text { fraction } \kappa\end{array}$ & $\begin{array}{c}\text { Launch mass, } \\
\mathrm{kg}\end{array}$ & $\begin{array}{c}\text { Side length, } \\
\mathrm{m}\end{array}$ \\
\hline 1 & 0.871 & 459.2 & 243 \\
2 & 0.742 & 539.1 & 264 \\
3 & 0.613 & 652.5 & 290 \\
3.56 & 0.541 & 739.4 & 309 \\
4 & 0.484 & 826.4 & 327 \\
5 & 0.355 & 1126.8 & 381 \\
\hline \hline
\end{tabular}

digital control unit, three engine gimbals, and xenon tank/feedlines amount to $360 \mathrm{~kg}$ with the solar array included. Thus, the mass of the spacecraft, without propulsion, is $348 \mathrm{~kg}$. To adapt this spacecraft to other propulsion methods, a small solar array must be added for electrical power. For example, NEAR-Shoemaker solar array mass was $46.1 \mathrm{~kg}$, so that it is assumed that a propulsion nonspecific Dawn spacecraft weighs on the order of $400 \mathrm{~kg}$. Main-belt asteroid missions are likely to require larger solar arrays due to the increased distance from the sun. This mission has been reconfigured to utilize solar sail propulsion, for the same Earth launch date of 27 May 2006 and 11-month orbiter stay times at each asteroid. The trajectories are shown in Fig. 9 for a sail characteristic acceleration of $1.0 \mathrm{~mm} \cdot \mathrm{s}^{-2}$. The Earth-Vesta phase was 3.2 years and the Vesta-Ceres phase lasted 3.7 years. With use of a more modest characteristic acceleration of $0.52 \mathrm{~mm} / \mathrm{s}^{2}$, the sail would take 4.9 years to reach Vesta, but the interasteroid phases would be prolonged due to the diminished solar photon pressure out at the main-belt distance from the sun. Sail sizing was conducted for a $1.0-\mathrm{mm} \cdot \mathrm{s}^{-2}$ sail with the Dawn payload and is shown in Table 9. It was discovered that if a sail assembly loading of $3.56 \mathrm{~g} \cdot \mathrm{m}^{-2}$ were utilized, the launch mass could be reduced by $33 \%$ to around $740 \mathrm{~kg}$. The solar sail component would be of mass $340 \mathrm{~kg}$ and of square side length $309 \mathrm{~m}$. If a high-performance sail such as this could not be fabricated, or the sail dimensions were problematically large, perhaps some payload and bus miniturization could be accomplished. Table 10 shows the launch masses and sail side lengths for lower performance sails with reduced payload masses. We have extended the mission objectives from Vesta and Ceres to two further asteroids, Lucina and Lutetia, to further demonstrate the viability of using a solar sail in a main-belt asteroid survey scenario. The trajectories from Ceres to Lucina to Lutetia are shown in Fig. 10 for $1.0 \mathrm{~mm} \cdot \mathrm{s}^{-2}$, again with 11-month stay times at each asteroid.

Table 10 Sailcraft sizing for $1.0-\mathrm{mm} \cdot \mathrm{s}^{-2}$ asteroid rendezvous mission with reduced payload masses

\begin{tabular}{lcccc}
\hline \hline \multirow{2}{*}{$\begin{array}{l}\text { Sail assembly } \\
\text { loading } \sigma_{s}, \mathrm{~g} \cdot \mathrm{m}^{-2}\end{array}$} & $\begin{array}{c}\text { Payload mass } \\
\text { fraction } \kappa\end{array}$ & \multicolumn{3}{c}{ Launch mass, kg; sail side length, m } \\
\cline { 3 - 5 } & 0.355 & $282 ; 191$ & $563 ; 270$ & $845 ; 330$ \\
5 & 0.226 & $442 ; 239$ & $885 ; 338$ & $1327 ; 414$ \\
6 & 0.097 & $1031 ; 365$ & $2062 ; 516$ & $3093 ; 632$ \\
\hline
\end{tabular}

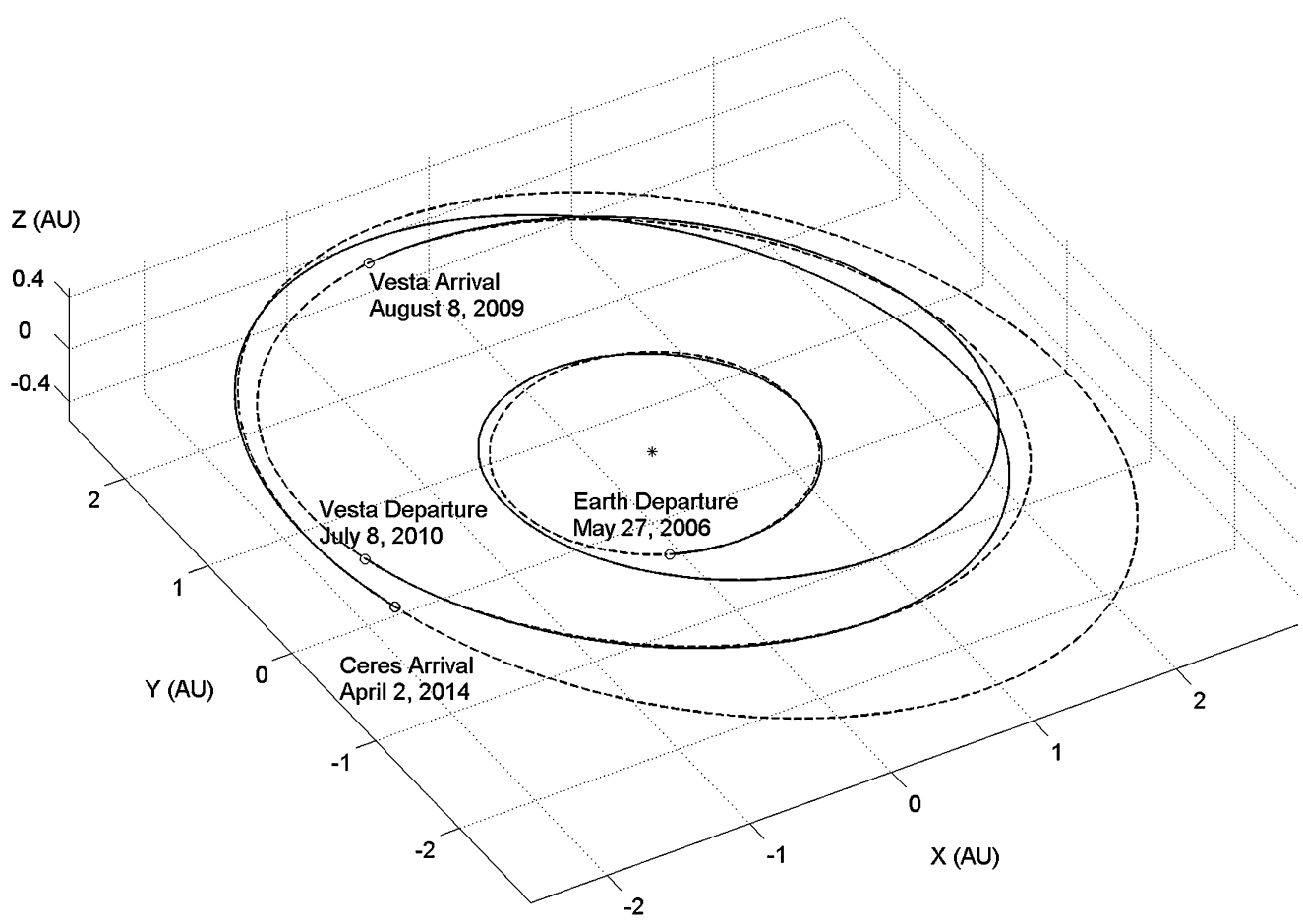

Fig. 9 Earth-Vesta-Ceres rendezvous. 


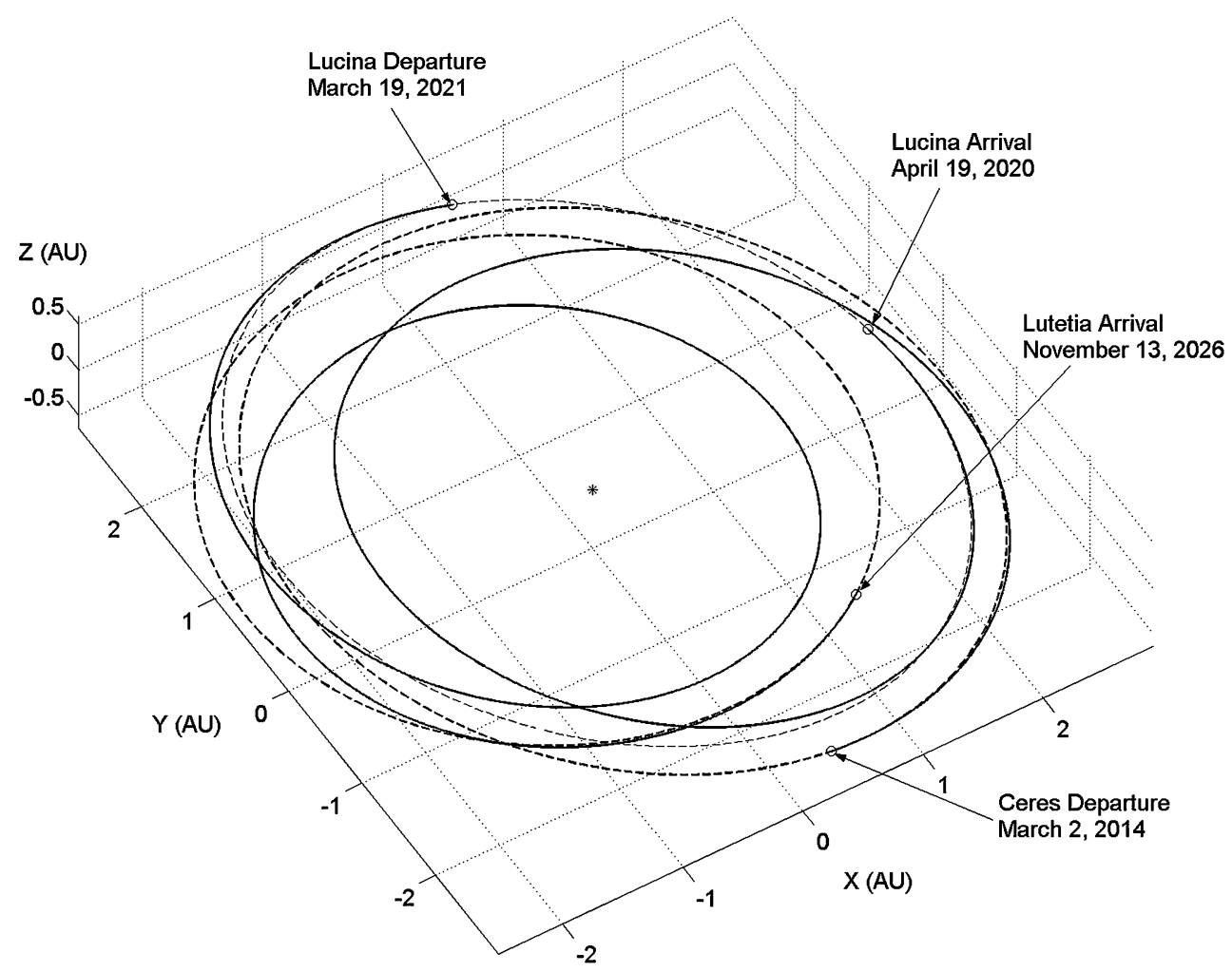

Fig. 10 Ceres-Lucina-Lutetia rendezvous.

\section{Conclusions}

SPC rendezvous missions have been shown to be an attractive application of solar sail propulsion due to their high-energy requirements. The original Rosetta mission to Wirtanen could have been enabled using current solar sail technology, with a large enough sail, that would have resulted in a $68 \%$ reduction in trip time and a $44 \%$ reduction in launch mass, greatly reducing the mission cost. Previous examples of newly discovered LPCs such as Hale-Bopp and others have been adopted, and fast flyby intercept missions are seen to be feasible providing investment is made into developing the associated enabling technologies. Intercept is rapidly possible at perihelion, with high-performance sailcraft, or at orbit node, with more modest technology. The option of Earth return means that dust sample return is possible, although further research would be necessary to ascertain whether the sail film could survive such a high-speed dust encounter. Multiple comet flyby scenarios would maximize science return if the scientific payload were minimal, to reduce sail dimensions. Multiple hyperbolic comet flybys can be achieved due to the sail's unlimited $\Delta V$ capability and, with a close solar pass, could open up potential for rapid investigation of the outer solar system and the interstellar medium. A sailcraft with a low characteristic acceleration of $0.362 \mathrm{~mm} \cdot \mathrm{s}^{-2}$ would have enabled a nodal flypast of Hale-Bopp with Earth orbit departure following first discovery. For a science payload of $50 \mathrm{~kg}$ and an assembly loading of $15 \mathrm{~g} \cdot \mathrm{m}^{-2}$, a square sail of side $89 \mathrm{~m}$ could be delivered to GEO, along with a commercial satellite, as an auxiliary payload on a Delta 4 class launcher. This demonstrates the potential, given technology readiness, of rapid solar sail interception of a new LPC following its initial apparition. Solar sails could open up vast opportunities for multiple asteroid survey missions. Replacing the solar electric propulsion component of the Dawn mission with a solar sail that can obtain equivalent trip times could result in a $33 \%$ reduction in launch mass and, hence, cost. It was shown that a unique advantage of using a solar sail would be to extend the mission objectives to orbit two further asteroids. The open-ended nature of solar sail propulsion has again been demonstrated, and in theory, many more asteroids could be visited. The fundamental point to stress for all of the mission scenarios presented is that solar sails do not rely on onboard propellant mass and, thus, significant launch mass savings can be realized, reducing mission cost as well as extending useful mission lifetime.

\section{Acknowledgments}

The Earth centered escape spirals were generated by Malcolm Macdonald at the University of Glasgow. The genetic algorithm used was developed by David L. Carroll at the University of Illinois, Urbana-Champaign. Thanks to James. E. Rogan, Program Manager, Team Encounter LLC, Houston, Texas, for updates on sail assembly loadings.

\section{References}

${ }^{1}$ Sims, J. A., "Trajectories to Comets Using Solar Electric Propulsion," American Astronautical Society, AAS Paper 00-134, Jan. 2000.

${ }^{2}$ Perozzi, E., and Fabiani, V., "On Targeting Long-Period and New Comets for Small Satellite Missions," Proceedings of the Workshop on Space Exploration and Resources Exploitation, ESA Publ. ESA-WPP-151, preprint, 1998.

${ }^{3}$ Wright, J. L., Space Sailing, 1st ed., Gordon and Breach Science, Montreux, Switzerland, 1992, pp. 42-44.

${ }^{4}$ Kluever, C. A., "Comet Rendezvous Mission Design Using Solar Electric Propulsion Spacecraft," Journal of Spacecraft and Rockets, Vol. 37, No. 5, 2000, pp. 698-700.

${ }^{5}$ Królikowska, M., "A Study of the Original Orbits of hyperbolic Comets," Astronomy and Astrophysics, Vol. 376, No. 1, 2001, pp. 316-324.

${ }^{6}$ Perozzi, E., Rondinelli, G., Di Genova, G., Pittich, E. M., and Valsecchi, G. B., "Small Satellite Missions to Long-Period Comets: The Hale-Bopp Opportunity," Acta Astronautica, Vol. 39, Nos. 1-4, 1996, pp. 45-50.

Leipold, M. E., "Solar Sail Mission Design," Ph.D. Dissertation, Dept. of Flight Mechanics and Flight Control, Technical Univ. of Munich, Munich, Feb. 2000

${ }^{8}$ Colasurdo, G., and, Casalino, L., "Missions to Asteroids Using Solar Electric Propulsion," Acta Astronautica, Vol. 50, No. 11, 2002, pp. 705-711.

${ }^{9}$ Yen, C. L., "Comparing Solar Sail and Solar Electric Propulsion for Propulsive Effectiveness in Deep Space Missions," American Astronautical Society, AAS Paper 01-214, Feb. 2001

${ }^{10}$ Morrow, E., Scheeres, D. J., and Lubin, D., "Solar Sail Orbit Operations at Asteroids," Journal of Spacecraft and Rockets, Vol. 38, No. 2, 2001, pp. 279-286. 
${ }^{11}$ Morrow, E. M., “Solar Sail Orbit Operations," Ph.D. Dissertation, Dept. of Aerospace Engineering, Univ. of California, San Diego, CA, 2002.

${ }^{12}$ McInnes, C. R., Solar Sailing: Technology, Dynamics and Mission Applications, 1st ed., Springer-Praxis Series in Space Science and Technology, Springer-Verlag, Berlin, 1999, p. 115.

${ }^{13}$ Sauer, C. G., Jr., "Optimum Solar Sail Interplanetary Trajectories," American Astronautical Society, AAS Paper 76-792, Aug. 1976.

${ }^{14}$ Forward, R. L., "Statite: A Spacecraft That Does Not Orbit," Journal of Spacecraft and Rockets, Vol. 28, No. 5, 1991, pp. 606-611.

${ }^{15}$ McInnes, C. R., Hughes, G. W., and Macdonald, M., "Low-Cost Mercury Orbiter and Sample Return Missions Using Solar Sail Propulsion," Aeronautical Journal, Aug. 2003, pp. 469-478.

${ }^{16}$ Hughes, G. W., and McInnes, C. R., "Solar Sail Hybrid Trajectory Optimization for Non-Keplerian Orbit Transfers," Journal of Guidance, Control, and Dynamics, Vol. 25, No. 3, 2002, pp. 602-604.

${ }^{17}$ Rauwolf, G. A., and Friedlander, A., "Near-Optimal Solar Sail Trajectories Generated by a Genetic Algorithm," American Astronautical Society, AAS Paper 99-332, Aug. 1999.

${ }^{18}$ Bader, J. L., "Optimal Low-Thrust Out-of-Ecliptic Trajectories," Dept. of Mechanical and Aerospace Engineering, MAE 502 Final Project Rept., Princeton Univ., Princeton, NJ, Jan. 2002.
${ }^{19}$ Walker, M. J. H., Ireland, B., and Owens, J., "A Set of Modified Equinoctial Orbit Elements," Celestial Mechanics, Vol. 36, 1985, pp. 409-419.

${ }^{20}$ Sauer, C. G., Jr., "Solar Sail Trajectories for Solar-Polar and Interstellar Probe Missions," American Astronautical Society, AAS Paper 99-336, Aug. 1999.

${ }^{21}$ Cohen, D., Gloyer, P., and Rogan, J., "Preliminary Design of a High Performance Solar Sailing Mission," 16th Annual AIAA/Utah State Univ. Conf. on Small Satellites, Paper SSC02-II-5, Aug. 2002.

${ }^{22}$ Macdonald, M., and McInnes, C. R., "Analytical Control Laws for NearOptimal Geocentric Solar Sail Transfers," American Astronautical Society, AAS Paper 01-472, July-Aug. 2001.

${ }^{23}$ Russell, C. T., Coradini, A., Feldman, W. C., Jaumann, R., Konopliv, A. S., McCord, T. B., McFadden, L. A., McSween, H. Y., Motolla, S., Neukum, G., Pieters, C. M., Raymond, C. A., Smith, D. E., Sykes, M. V., Williams, B. G., and Zuber, M. T., "Dawn: A Journey to the Beginning of the Solar System," Asteroids, Comets, Meteors Conference Proceedings, ESA Publ. SP-500, edited by B. Warmbein, Berlin, 2002. 